Check for updates

Cite this: J. Mater. Chem. C, 2021 9, 1362

Received 4th September 2020, Accepted 21st December 2020

DOI: $10.1039 / \mathrm{d} 0 \mathrm{tc} 04225 \mathrm{k}$

rsc.li/materials-c

\title{
A quantum dynamics study of the hyperfluorescence mechanism $\uparrow$
}

\author{
Yvelin Giret, (D) Julien Eng, (D) Thomas Pope and Thomas Penfold (D)*
}

\begin{abstract}
Triplet state harvesting using thermally-activated delayed fluorescence (TADF) combined with efficient Förster resonant energy transfer (FRET) to a narrow fluorescent emitter is seen as a promising approach to achieve high efficiency and colour-purity in organic light-emitting diodes (OLEDs). In this work, we perform quantum chemistry and quantum dynamics simulations to model the so-called hyperfluorescence (HF) process between a carbene-metal-amide (CMA) molecule with a Au bridging metal (Au-Cz) and a narrow blue fluorescent emitter, 2,5,8,11-tetra-tert-butylperylene (TBPe). Our quantum dynamics simulations illustrate a FRET rate of $\sim 10^{10} \mathrm{~s}^{-1}$ indicating that it occurs on the picosecond timescale comparable with the ISC crossing rate of $\mathbf{A u}-\mathbf{C z}$. This high FRET rate, which is most strongly dependent on the energy difference between the $S_{1}$ states of the donor and acceptor molecules, is advantageous for devices as it encourages rapid triplet harvesting. In addition, the comparable FRET and intersystem crossing (ISC) rates, in contrast to most organic only systems, would facilitate studying this mechanism using photoexcitation. Besides the FRET rate, Förster radii are also estimated from the quantum dynamics simulations for different energy differences between the donor and acceptor molecules and are in quantitative agreement with the experimental estimations for different systems, showing that quantum nuclear dynamics simulation could be an important tool for enhancing our understanding of hyperfluorescence-based emitters.
\end{abstract}

\section{Introduction}

In the last decades there has been an intensive research effort conducted on developing and improving organic lightemitting diodes (OLEDs). ${ }^{1-3}$ The 1st generation of OLEDs were based on fluorescent molecules. However, these devices suffer from low internal quantum efficiency (IQE $<25 \%$ ), as they are unable to harvest the non-emissive triplet states generated by charge recombination. This can be somewhat improved by exploiting inefficient triplet annihilation, but they still remain well short of $100 \% \mathrm{IQE}^{4}$ Full triplet harvesting was first achieved using iridium or platinum containing phosphorescent emitters, which exploits the large intrinsic spin orbit coupling (SOC) of these metals to harvest the triplet states. $^{5}$

Alternatively, in 2009, Adachi and coworkers proposed an OLED architecture based upon purely organic molecules exploiting thermally-activated delayed fluorescence (TADF). ${ }^{6}$ This mechanism relies on a triplet state being thermally

Chemistry-School of Natural and Environmental Sciences, Newcastle University, Newcastle Upon Tyne, NE1 7RU, UK. E-mail: tom.penfold@newcastle.ac.uk $\dagger$ Electronic supplementary information (ESI) available. See DOI: 10.1039/ dotc04225k activated to become iso-energetic with a singlet excited state such that it can undergo reverse intersystem crossing (rISC) to the singlet manifold. For molecules with small singlet-triplet energy gap TADF can become very efficient allowing a near $100 \%$ internal quantum efficiency without the use of rare earth elements. ${ }^{7-12}$ However, while able to achieve 100\% IQE, both phosphorescence and TADF OLED emitters usually require the use of charge-transfer (CT) excited states. These CT states have inherently broad emission, with a typical full-width at halfmaximum (FWHM) 70-120 nm, reducing the colour purity, making them difficult to use in displays, which require a FWHM $<30 \mathrm{~nm}$. Consequently, commercial OLED displays employ lossy colour filters and/or expensive, difficult to fabricate optical microcavity structures to achieve sufficiently narrow line widths to satisfy colour requirements. This filtering of the original electroluminescence (EL) significantly reduces the external quantum efficiency (EQE) of the display, increases power consumption and shortens operational lifetime because the pixels need to run at higher brightness to compensate for this loss.

To overcome this, Nakanotani et al. ${ }^{13}$ proposed co-depositing a highly luminescent fluorophore into the emissive layer of a TADF-based OLEDs. The aim is to combine the advantages associated with efficient triplet harvesting using TADF emitters 
a)

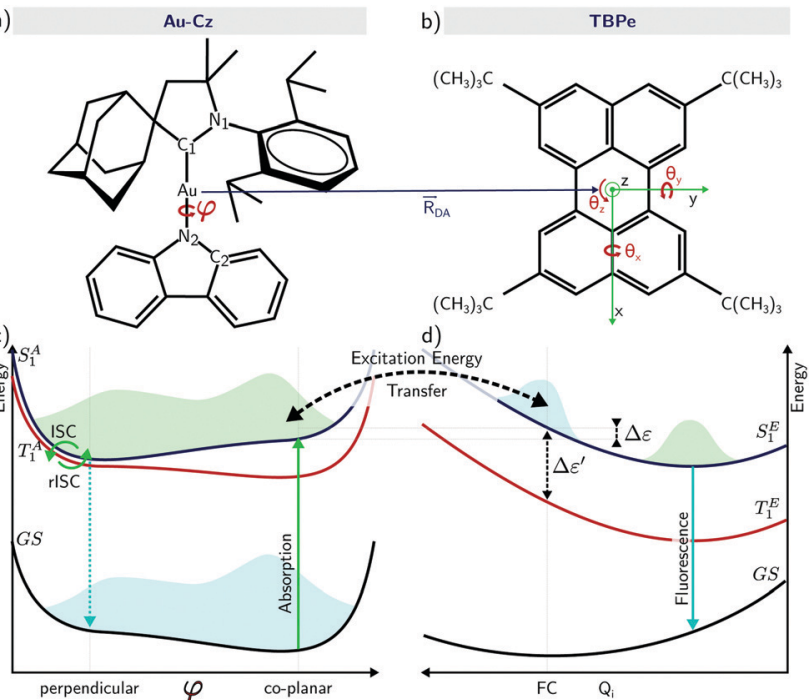

Fig. 1 A schematic of a Au-Cz-TBPe system used to investigate the hyperfluorescence mechanism. (a) The structure of the carbene-metalamide (CMA) molecule $\mathbf{A u - C z}$ including the $\varphi$ torsional degree of freedom important in the triplet harvesting. (b) The structure of TBPe including the distances and angles used relative to $\mathbf{A u - C z}$ to calculate the energy transfer coupling. ( $c$ and d) Schematics of the ground and lowest singlet and triplet potentials of $\mathbf{A u - C z}$ and TBPe involved in energy transfer. $\Delta \varepsilon$ is the energy difference between the $S_{1}$ states of the donor an acceptor molecule and $\Delta \varepsilon^{\prime}$ is the energy difference between the singlet and triplet states of TBPe.

and the narrow emission of rigid fluorescent organic molecules. In their approach, refereed to as hyperfluorescence (HF) and illustrated in Fig. 1, charge recombination occurs on the TADF emitter which performs rISC to the singlet state, and then undergoes Förster energy transfer to the rigid fluorescent molecules which emits with high efficiency and colour purity. Using this approach the authors were able to demonstrate narrow fluorescence-based OLED achieving external quantum efficiencies as high as $18 \%$ for blue, green, yellow and red emission. In this study, the authors discussed the two regimes of HF accessed by either optical and electrical excitation. The latter is obviously more relevant in the context of device operation while the former is important in the context of material characterisation. Upon optical excitation, the authors reported that the excitons mainly formed on the host $\left(\mathrm{S}_{1}^{\mathrm{H}}\right)$ and are resonantly transferred to the TADF-assistant molecule $\left(S_{1}^{A}\right)$. From $S_{1}^{A}$, there is energy transfer into the emissive fluorescent molecule via FRET $\left(S_{1}^{\mathrm{E}}\right)$, giving rise to prompt fluorescence. Alternatively, ISC to the $\mathrm{T}_{1}^{\mathrm{A}}$ and then back to $\mathrm{S}_{1}^{\mathrm{A}}$ by rISC followed by FRET to $S_{1}^{\mathrm{E}}$ gives rise to delayed fluorescence. The relative importance of both pathways depends on the rate of FRET compared to the ISC rate but for most organic molecules studied to date the former dominates, meaning there is limited triplet formation and therefore ISC/rISC. For electrical excitation, excitons are directly formed on the TADF-assistant molecule with a ratio of $25 / 75 \%$ for $S_{1}^{A}$ and $T_{1}^{A}$ respectively, in which case they observed a significantly larger contribution of the delayed fluorescence in the total emission.
In the HF approach, the fluorescence emitter can be chosen or designed ${ }^{14,15}$ to exhibit both a large oscillator strength, which minimises the excited state lifetime, and a narrow emission to increase the colour purity of the device. In addition, it has been shown that it is important to suppress Dexter energy transfer from the triplet states of TADF to the fluorophore and this can be achieved by either controlling the concentration of the TADF co-host ${ }^{16}$ or using Dendritic Fluorophores. ${ }^{17,18}$ In both cases, suppression of Dexter energy transfer can be achieved by increasing the spatial separation between the TADF co-host and the fluorophore.

To provide a deeper understanding into the HF mechanism, kinetic Monte Carlo (KMC) simulations were used to demonstrate that the IQE can be enhanced by aligning the appropriate energy levels and avoiding the formation of charge-transfer excitons, where the electron (or hole) is trapped on the TADF co-host and the other charge on the fluorophore. ${ }^{19}$ For the TADF co-host to be efficient, a small $\Delta E_{\mathrm{S}_{1}-\mathrm{T}_{1}}$ is required, however as widely discussed in the TADF literature, ${ }^{11,12}$ when achieved with $\mathrm{D}-\mathrm{A}$ molecules this reduces the radiative rate of the $S_{1}$ state. The consequence of this in the framework of the HF mechanism would be to suppress the Förster energy transfer rate to the fluorophore. From this perspective, Lyskov et al. ${ }^{20}$ demonstrated that molecular vibrations and molecular orientation of the TADF co-host were important to increase the rate of the exciton transfer, the latter being similar to the well established spin-vibronic mechanism for TADF. ${ }^{21-23}$

In the vast majority of cases to date, HF devices have used organic TADF co-hosts. However, recently Yang et al. ${ }^{24}$ demonstrated high performance OLED with EQEs $>20 \%$ using energy transfer from a carbene metal-amide (CMA) TADF co-host, where the bridging atom was $\mathrm{Au}$ (Au-Cz). Ultrafast optical measurements were used to propose that interfluorophore energy transfer occurs with efficiency $>95 \%$ within 300 fs. $\mathbf{A u}-\mathbf{C z}$ has previously been shown to exhibit rapid singlet-triplet interconversion and high performance in OLED devices ${ }^{25}$ leading to a significant interest in developing this framework of emitters. ${ }^{26-31}$ On the basis of timeresolved electroluminescence (EL) and photoluminescence (PL) measurements, Di et al. demonstrated for $\mathbf{A u}-\mathbf{C z}$ rapid ISC to triplets $(\sim 4 \mathrm{ps})$, and that CMA emission occurs almost entirely via a delayed-emission channel ( $350 \mathrm{~ns}) .{ }^{25}$ Importantly, the torsional motion around the bridge brings the Au-Cz to a (perpendicular) configuration showing a very small energy gap between the $S_{1}$ and $T_{1}$ states and very small oscillator strength, while the (co-planar) configuration shows a higher energy gap but also a higher oscillator strength. In other words, the co-planar geometry will promote emission and FRET while the perpendicular geometry will promote rISC. ${ }^{10,32,33}$

In this paper, we combine quantum chemistry and quantum dynamics calculations to provide a detailed understanding of the excited state properties required for efficient HF. We find a FRET rate of $\sim 10^{10} \mathrm{~s}^{-1}$ which is comparable with the ISC crossing rate of Au-Cz. This rate is most strongly dependent on 
the energy between the $S_{1}$ states of the donor and acceptor molecules. Besides the FRET rate, Förster radii are also estimated from the quantum dynamics simulations and are in quantitative agreement with the experimental estimations for different systems.

\section{Methodology}

\subsection{Computational details}

All quantum chemistry calculations were performed using the QChem 5.0 quantum chemistry package ${ }^{34}$ with the exception of the spin-orbit coupling matrix elements which are calculated with the Amsterdam Modelling Suite (ADF) (2014). ${ }^{35,36}$ Density functional theory (DFT) and time-dependent density functional theory (TDDFT) were used to optimise the geometry of the ground state and the excited states, respectively. We used for all atoms all-electron Gaussian basis sets of triple zeta valence quality (def2-TZVP), ${ }^{37}$ except for the Au atom where an effective core potential (ECP) is used. ${ }^{38}$ All calculations are performed at the hybrid level with PBE0 exchange-correlation functional, ${ }^{39}$ and the TDDFT calculations within the Tamm-Dancoff approximation (TDA). ${ }^{40}$ Finally, calculations were performed with an implicit solvent model within the conductor-like screening model (COSMO) approach ${ }^{41}$ with the properties of toluene.

\subsection{Nuclear wavepacket propagation and model Hamiltonian}

Throughout this study our quantum dynamics simulations were based upon a model Hamiltonian for Au-Cz and TBPe with the structure:

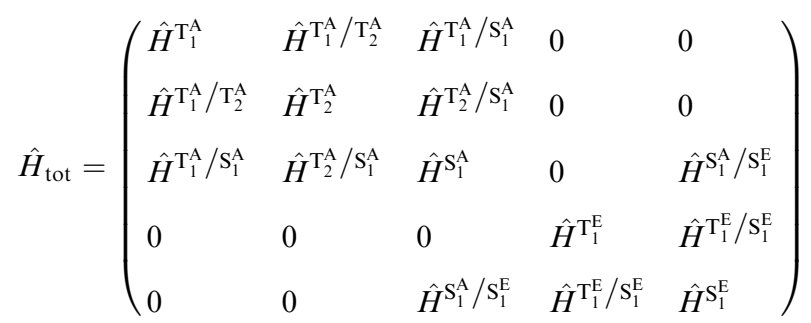

here the superscript A refers to the TADF assistant dopant $(\mathbf{A u}-\mathbf{C z})$, and the superscript E refers to the fluorescent emitter (TBPe). The upper-left $3 \times 3$ block matrix corresponds to the Au-Cz Hamiltonian, ${ }^{32,33}$ the lower-right $2 \times 2$ block matrix corresponds to the TBPe Hamiltonian written within the linearvibronic coupling (LVC) model. ${ }^{21} \hat{H}^{\mathrm{S}_{1}^{\mathrm{A}} / \mathrm{S}_{1}^{\mathrm{E}}}$ is the Hamiltonian describing the coupling between the singlet states of the two molecules. Full details about the calculation of the Hamiltonian matrix elements are given in the ESI. $\dagger$ In brief, the intermolecular coupling Hamiltonian will describe the excited state energy transfer (EET) process between singlet states of CMA $\left(\mathrm{S}_{1}^{\mathrm{A}}\right)$ and TBPe $\left(S_{1}^{\mathrm{E}}\right)$. After a single-excitation calculation at the TDDFT(PBE0)/TDA level, the coupling values are calculated with the fragment-excitation difference (FED) method, ${ }^{42}$ as implemented in the QChem package. EET, FED, and Förster theory are briefly presented in the ESI. $\dagger$ In addition to the 4 vibrational modes of the TBPe molecule described in the ESI, $\dagger$ the $\mathbf{A u}-\mathbf{C z}$ molecule is described by 2 degrees of freedom, namely, the torsion around the $\mathrm{Au}-\mathrm{N}$ bond $(\varphi)$ and the stretching mode of the same bond $\left(r_{\mathrm{Au}-\mathrm{N}}\right)$. We refer the reader to ref. 32 and 33 for a full description of this model Hamiltonian. Finally, we define the distance $\left(R_{\mathrm{DA}}\right)$ as the distance between the $\mathrm{Au}$ atom in CMA and the center of mass (COM) of TBPe, as shown in Fig. 1.

The quantum dynamics were performed using the multiconfigurational time-dependent Hartree (MCTDH) method as implemented within the Quantics quantum dynamics package. $^{43}$ The 2-dimensional spin-vibronic Hamiltonian model for the TADF emitter (Au-Cz molecule) was represented on a Fast Fourier Transform (FFT) grid of $1152 \times 864$ grid points for the torsion and the stretching modes, and where the torsional mode was set with periodic boundary conditions ranging from $-\pi \rightarrow \pi .^{32,33}$ For the torsion and the stretching modes, 7 single particle functions (SPFs) were used for $\mathrm{T}_{1}^{\mathrm{A}}$ and $\mathrm{S}_{1}^{\mathrm{A}}$, and 2 for $\mathrm{T}_{2}^{\mathrm{A}}, \mathrm{S}_{1}^{\mathrm{E}}$ and $\mathrm{T}_{1}^{\mathrm{E}}$. The four normal modes of TBPe were described using the multi-set formalism and combined in two pairs. 7 SPFs were used for $\mathrm{S}_{1}^{\mathrm{E}}$ and $\mathrm{T}_{1}^{\mathrm{E}}$, and 2 SPFs were used for all the states of Au-Cz. The evolution of the $A$-vector was calculated with the constant mean field integration scheme, and the SPFs were propagated with the Runge-Kutta integrator to fifth order (RK5). The initial wavepacket is placed onto the $S_{1}^{A}$ surface of Au-Cz. To better represent the distribution of $\varphi$ associated with the flat nature of the potential along this motion, a wavepacket with a larger width along the torsional mode was adopted.

\subsection{Kinetic model}

To interpret the results from the quantum dynamics and extract rate constants, we have built a kinetic model with three states, namely, $\mathrm{S}_{1}^{\mathrm{A}}, \mathrm{S}_{1}^{\mathrm{E}}$, and $\mathrm{T}_{1}^{\mathrm{A}}$, and their interactions, namely, the population transfer from $S_{1}^{A}$ to $T_{1}^{A}$ (ISC), from $T_{1}^{A}$ to $S_{1}^{A}$ (rISC), from $S_{1}^{\mathrm{A}}$ to $S_{1}^{\mathrm{E}}(\mathrm{EET})$, and from $S_{1}^{\mathrm{E}}$ to $S_{1}^{\mathrm{A}}$ (rEET). The kinetics of the population transfer are described by:

$$
\begin{aligned}
& \frac{\mathrm{dS}_{1}^{\mathrm{A}}(t)}{\mathrm{d} t}=-\left(k_{\mathrm{ISC}}+k_{\mathrm{EET}}\right) \cdot \mathrm{S}_{1}^{\mathrm{A}}(t)+k_{\mathrm{rISC}} \cdot \mathrm{T}_{1}^{\mathrm{A}}(t)+k_{\mathrm{rEET}} \cdot \mathrm{S}_{1}^{\mathrm{E}}(t) \\
& \frac{\mathrm{dT}_{1}^{\mathrm{A}}(t)}{\mathrm{d} t}=-k_{\mathrm{rISC}} \cdot \mathrm{T}_{1}^{\mathrm{A}}(t)+k_{\mathrm{ISC}} \cdot \mathrm{S}_{1}^{\mathrm{A}}(t) \\
& \frac{\mathrm{d} \mathrm{S}_{1}^{\mathrm{E}}(t)}{\mathrm{d} t}=-k_{\mathrm{rEET}} \cdot \mathrm{S}_{1}^{\mathrm{E}}(t)+k_{\mathrm{EET}} \cdot \mathrm{S}_{1}^{\mathrm{A}}(t)
\end{aligned}
$$

where $k_{\text {ISC }}$ is the ISC rate constant from $\mathrm{S}_{1}^{\mathrm{A}}$ to $\mathrm{T}_{1}^{\mathrm{A}}, k_{\mathrm{rISC}}$ the rISC rate constant from $\mathrm{T}_{1}^{\mathrm{A}}$ to $\mathrm{S}_{1}^{\mathrm{A}}, k_{\mathrm{EET}}$ the EET rate constant from $\mathrm{S}_{1}^{\mathrm{A}}$ to $S_{1}^{\mathrm{E}}$, and $k_{\text {rEET }}$ the reverse EET rate constant from $S_{1}^{\mathrm{E}}$ to $S_{1}^{\mathrm{A}}$. Resolution of equation system (2) for the general initial conditions $\mathrm{S}_{1}^{\mathrm{A}}(0)=n, \mathrm{~T}_{1}^{\mathrm{A}}(0)=1-n$, and $\mathrm{S}_{1}^{\mathrm{E}}(0)=0$ is given in the ESI. $\dagger$ For the initial conditions $\mathrm{S}_{1}^{\mathrm{A}}(0)=1, \mathrm{~T}_{1}^{\mathrm{A}}(0)=0$, and $\mathrm{S}_{1}^{\mathrm{E}}(0)=0$, which corresponds to an optical excitation on the $\mathbf{A u}-\mathbf{C z}$ 
molecule, solutions are given by:

$$
\begin{aligned}
\mathrm{S}_{1}^{\mathrm{A}}(t)=\gamma_{1}+ & {\left[\left(2(b+d)-\left(1+\gamma_{1}\right) \sigma_{1}\right) \frac{\sinh \left(\frac{\theta_{1} t}{2}\right)}{\theta_{1}}\right.} \\
& \left.+\left(1-\gamma_{1}\right) \cosh \left(\frac{\theta_{1} t}{2}\right)\right] \mathrm{e}^{-\sigma_{1} t / 2} \\
\mathrm{~T}_{1}^{\mathrm{A}}(t)=\gamma_{2}+ & {\left[\begin{array}{l}
\left.\left(2 a-\gamma_{2} \sigma_{1}\right) \frac{\sinh \left(\frac{\theta_{1} t}{2}\right)}{\theta_{1}}-\gamma_{2} \cosh \left(\frac{\theta_{1} t}{2}\right)\right] \mathrm{e}^{-\sigma_{1} t / 2} \\
\mathrm{~S}_{1}^{\mathrm{E}}(t)=\gamma_{3}+
\end{array}\right.} \\
& {\left[\left(2 c-\gamma_{3} \sigma_{1}\right) \frac{\sinh \left(\frac{\theta_{1} t}{2}\right)}{\theta_{1}}-\gamma_{3} \cosh \left(\frac{\theta_{1} t}{2}\right)\right] \mathrm{e}^{-\sigma_{1} t / 2} . }
\end{aligned}
$$

where $a=k_{\mathrm{ISC}}, b=k_{\mathrm{rISC}}, c=k_{\mathrm{EET}}, d=k_{\mathrm{rEET}}, \sigma_{1}=a+b+c+d, \sigma_{2}=$ $a d+b c+b d, \theta_{1}=\sqrt{\sigma_{1}^{2}-4 \sigma_{2}}, \gamma_{1}=\frac{b d}{\sigma_{2}}, \gamma_{2}=\frac{a d}{\sigma_{2}}$, and $\gamma_{3}=\frac{b c}{\sigma_{2}} \cdot \sigma_{1}$ is defined as the sum of the first-order processes and $\sigma_{2}$ as the sum of the second-order processes. $\gamma_{i}$ represent the ratio of the second order processes populating each state, and $\theta_{1}$ represents a subtle competition between the different processes.

Throughout this work the radiative and non-radiative transitions of donor and acceptor molecules were not included as these channels were not included in the quantum dynamics and occur at longer timescales than considered within this work. Our model is a closed quantum system and therefore the kinetic model was setup to reflect the core processes present in the quantum dynamics. These equations above are used, with the rates $k_{\mathrm{ISC}}, k_{\mathrm{rISC}}$ and $k_{\mathrm{EET}}$, obtained by performing a fit to the population kinetics obtained from the quantum dynamics simulations.

\section{Results and discussion}

\subsection{Electronic structure}

The electronic structure of $\mathbf{A u}-\mathbf{C z}$ has previously been discussed in detail and therefore readers are referred to ref. 32 and 33 for further details. In the ground state geometry of TBPe, the four lowest excited state are: $\mathrm{T}_{1}$ with an excitation energy of $1.90 \mathrm{eV}$, $\mathrm{S}_{1}$ with an excitation energy of $3.03 \mathrm{eV}, \mathrm{T}_{2}$ with an excitation energy of $3.17 \mathrm{eV}$, and $\mathrm{T}_{3}$ with an excitation energy of $3.38 \mathrm{eV}$. As shown in Table $\mathrm{S} 1$ (ESI $\dagger$ ), the $\mathrm{T}_{1}$ and $\mathrm{S}_{1}$ states, which will be incorporated in the quantum dynamics, are both almost entirely composed of a HOMO $\rightarrow$ LUMO transition, and correspond to a local $\pi \rightarrow \pi^{*}$ excitation. To study the EET mechanism between the two molecules, the reference geometry is taken as Au-Cz at its $\mathrm{S}_{1}$ optimised geometry (perpendicular) and TBPe at its $\mathrm{S}_{0}$ optimised geometry, which is the starting configuration for the EET mechanism and corresponds to the geometry of the $S_{1}$ state of the whole system. The $S_{2}$ state of the whole system at this geometry corresponds to the $S_{1}$ state of the isolated TBPe molecule. The density differences between the excited states and the ground state for this reference
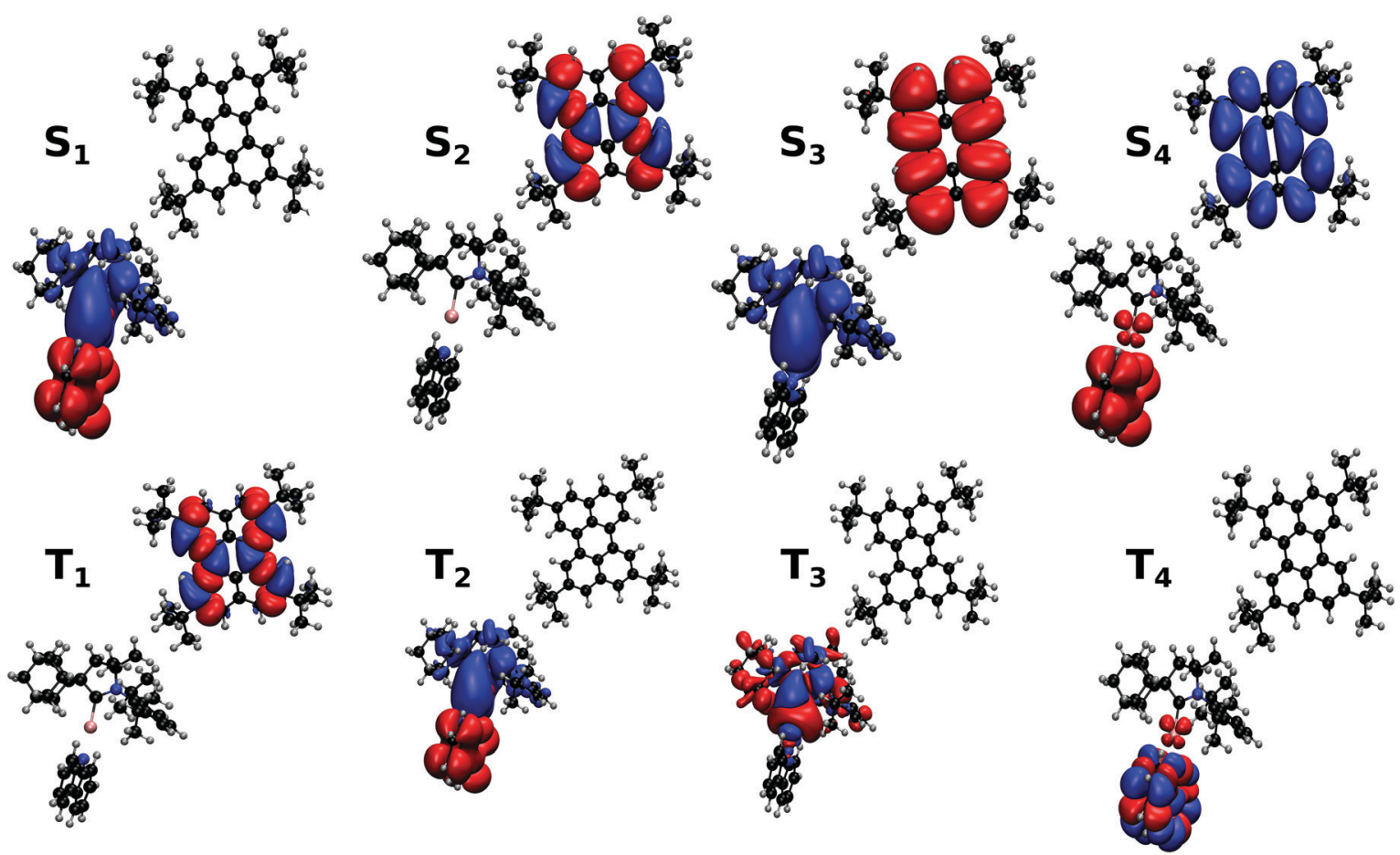

Fig. 2 Electronic density differences between excited states and the ground state with $\mathbf{A u}-\mathbf{C z}$ in the perpendicular geometry and TBPe for an intermolecular distance of $\sim 24.2 \AA$ and a random relative orientation (blue: increase, red: decrease). The singlet and triplet states shown are those of the whole system 
geometry at an intermolecular distance of $\sim 24.2 \AA$ are shown in Fig. 2. This also shows that the $\mathrm{S}_{3}$ and $\mathrm{S}_{4}$ states correspond to intermolecular CT states. Importantly, singlet-triplet conversation between these states $\left({ }^{1} \mathrm{CT}\right.$ and $\left.{ }^{3} \mathrm{CT}\right)$ was recently invoked as a significant loss pathway in HF-OLEDs. ${ }^{19}$ As shown in Fig. S1 (ESI $\dagger$ ), the energy of these states increases with intermolecular distance and for separations greater than $30 \AA$ the energy gap between these CT states and the $\mathrm{S}_{1}$ state is $0.6 \mathrm{eV}$. Given this energy gap, which is already likely to be underestimated due to the challenges of describe CT states with TDDFT, ${ }^{44}$ we do not include $S_{3}$ and $S_{4}$ states in the quantum dynamics. As a point of comparison, we have also calculated the electronic structure of Au-Cz-TBRb system. This was the combination of TADF co-dopant and fluorescence emitter recently used by Yang et al. in ref. 24, and generated narrow green emission in an OLED device. As shown in ESI, $\dagger$ this yields a similar electronic structure as the Au-Cz-TBPe system, albeit a slightly smaller EET coupling. Consequently, we focus on the Au-Cz-TBPe system as a way of generating a narrow blue HF setup.

We now turn to the EET coupling, responsible for the HF mechanism. This coupling depends on several key parameters, namely, the distance between the donor and acceptor $\left(R_{\mathrm{DA}}\right)$, the relative orientation of the two molecules described here by $\theta_{x}$, $\theta_{y}$ and $\theta_{z}$ and the Au-Cz dihedral angle $(\varphi)$ which has a large effect on the oscillator strength and the energy of the $S_{1}$ state of Au-Cz. ${ }^{32,33}$ Besides $\varphi$ we neglect the effect of all other intramolecular motions of either the Au-Cz or TBPe on the EET coupling. We first fix the relative orientation of the two molecules and calculate the coupling along $R_{\mathrm{DA}}$ for the perpendicular and the co-planar geometries, as shown by the blue stars in Fig. 3a and b. Moreover, we compare the EET coupling to the Förster coupling calculated from eqn (S44) (ESI $\dagger$ ) shown as red stars. As expected, the two coupling are in good agreement at larger distances $\left(R_{\mathrm{DA}} \geq 20 \AA\right)$, but deviate from each other at short distances, as discussed in the ESI. $\dagger$ Subsequently, we fix the distance $R_{\mathrm{DA}}$ at $\sim 24.2 \AA$ where the two couplings are in agreement and calculate the couplings as a function of $\varphi$, as shown in Fig. 3c. The energy difference between the $S_{1}$ and the $\mathrm{T}_{1}$ states of Au-Cz (black) is also plotted. This shows that it is possible to either have a small energy difference between the states, which exists for the perpendicular configuration of Au-Cz or a higher EET coupling, i.e. for the co-planar configuration. This arises because, as previously discussed, ${ }^{32,33}$ the dihedral angle $\varphi$ strongly controls the radiative rate of the $\mathrm{S}_{1}$ state. This highlights the fact that for the $\mathbf{A u}-\mathbf{C z}$ molecule to be efficient for HF, it needs to keep some freedom over $\varphi$ in order to harvest the triplets states in the perpendicular configuration and to transfer exciton energy to TBPe in the co-planar configuration.

Finally, we fix the position of Au-Cz in the perpendicular geometry and calculate the coupling along three inertia axis for TBPe $\left(\theta_{x}, \theta_{y}, \theta_{z}\right)$, as shown in Fig. S2 (ESI $\left.\dagger\right)$. Except for $\theta_{z}$ where the two couplings are slightly shifted, Förster coupling is systematically higher than the EET coupling. These differences can arise from multipolar Coulomb effects which are neglected in the Förster theory, as discussed in the ESI. $\dagger$ The influence of these angles on the EET is much less than $\varphi$ and therefore for the quantum dynamics, we only retain this dependences and fix the intermolecular distance at $\sim 15.7 \AA$. By doing so, $R_{\mathrm{DA}}$ is high enough to stay outside the short-range regime related to intermolecular CT states and small enough to have a sufficiently large coupling.

\subsection{The dynamics}

Fig. 4a shows a prototypical example of the population kinetics for the $S_{1}^{A}, T_{1}^{A}, T_{2}^{A}$, and $S_{1}^{E}$ states during the first $100 \mathrm{ps}$ after the initial excitation into the $\mathrm{S}_{1}^{\mathrm{A}}$ state for $\Delta \varepsilon \sim-50 \mathrm{meV}, \Delta \varepsilon$ being defined as the energy difference between the $S_{1}^{E}$ state in the co-planar geometry and the $\mathrm{S}_{1}^{\mathrm{A}}$ state in the ground state geometry of TBPe (see Fig. 1). $\Delta \varepsilon=0$ corresponds to the perfect resonance between the two states. For the first $\sim 20 \mathrm{ps,} \mathrm{we} \mathrm{see}$ clear oscillations between the $S_{1}^{A}$ and $T_{1}^{A}$ states, which corresponds to the wavepacket oscillating between the parallel and
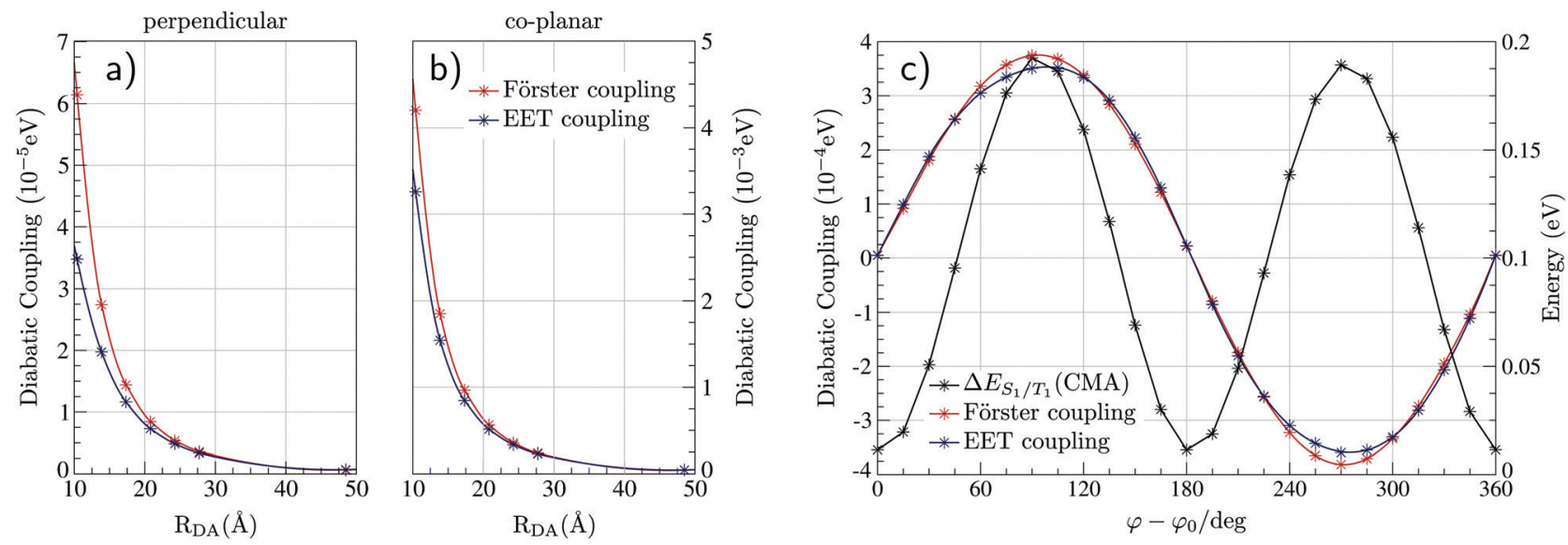

Fig. 3 Strength of the EET and the Förster couplings between $\mathbf{A u}-\mathbf{C z}$ and TBPe as a function of the intermolecular distance $R_{\mathrm{DA}}$ for the co-planar (a) and perpendicular geometry (b) of $\mathbf{A u}-\mathbf{C z}$. (c) Strength of the EET and the Förster couplings between $\mathbf{A u}-\mathbf{C z}$ and TBPe as a function of the torsion angle $\varphi$ of $\mathrm{Au}-\mathbf{C z}$ at $R_{\mathrm{DA}}=24.2 \AA$. This is also plotted with the $\mathrm{S}_{1}-\mathrm{T}_{1}$ energy gap between for $\mathbf{A u}-\mathbf{C z}$. Couplings as a function of $\theta_{x}, \theta_{y}$ and $\theta_{z}$ are shown in Fig. S2 $(\mathrm{ESI} \dagger)$. 
perpendicular geometries along the torsional angle potential of Au-Cz. ${ }^{32}$ On the other hand, the population in the $S_{1}^{E}$ state increases in a step-wise manner during the same duration and reaches a maximum of $\sim 20 \%$. This highlights the competition between the ISC/rISC process in the perpendicular geometry and the EET process in the parallel geometry, because increases in $S_{1}^{\mathrm{E}}$ are observed when $\mathbf{A u}-\mathbf{C z}$ is closer to the parallel geometry and ISC is unfavourable due to the larger energy gap between the singlet and triplet states. When fitted using the kinetic model outlined above this yields $k_{\mathrm{ISC}}=1.7 \times 10^{10} \mathrm{~s}^{-1}$ and $k_{\mathrm{F}}=$ $4.8 \times 10^{9} \mathrm{~s}^{-1}$. The former is in good agreement with previous work and experimental data, ${ }^{25,32}$ while the latter is consistent with a typical FRET rate.

Fig. $4 \mathrm{~b}$ shows the influence of $\Delta \varepsilon$ on the dynamics and in particular on the FRET rate, $k_{\mathrm{F}}$. As expected, the rate is largest when the two states are in resonance (i.e. $\Delta \varepsilon=0$ ). When $\Delta \varepsilon$ is negative $\left(\mathrm{S}_{1}^{\mathrm{A}}>\mathrm{S}_{1}^{\mathrm{E}}\right)$, the rate falls off slowly to $\sim 10^{9} \mathrm{~s}^{-1}$ at $\Delta \varepsilon=$ -0.5 . In contrast when $\Delta \varepsilon$ is positive $\left(S_{1}^{\mathrm{A}}<\mathrm{S}_{1}^{\mathrm{E}}\right)$ the rate falls off exponentially due to the introduction of an energy barrier required to promote FRET. At this point, it is noted that in all cases, except the resonant conditions, $k_{\mathrm{ISC}}>k_{\mathrm{EET}}$, this is advantageous for studying the HF mechanism using optical excitation, because in many systems (especially organic TADF emitters) $k_{\mathrm{ISC}}<k_{\mathrm{EET}}$ and therefore photoexcitation leads directly to EET without the ISC/rISC steps involved in HF occurring. Using the FRET rate, Fig. 4c shows the Förster radius calculated using our simulate $k_{\mathrm{F}}$ as a function of $\Delta \varepsilon$. These, with the exception of one point, are in quantitative agreement with experimental estimations for related and different systems, ${ }^{13,24}$ illustrating the potential for using the quantum dynamics to predict and refine the key properties of the components involved in HF.

Finally, we consider the potential interfering role of the $\mathrm{T}_{1}^{\mathrm{E}}$ state, indeed energy transfer into this state would be a detrimental loss pathway in the HF OLEDs. The calculated spinorbit coupling matrix elements between the $S_{1}^{\mathrm{E}}$ and $\mathrm{T}_{1}^{\mathrm{E}}$ states are very small $\left(\sim 10^{-10} \mathrm{eV}\right)$, and no contribution of the $\mathrm{T}_{1}^{\mathrm{E}}$ state appears in the population dynamics. To further investigate the possible role of spin-orbit coupling in the FRET mechanism, we artificially introduce a high coupling value $(\sim 5 \mathrm{meV})$ and increase the energy of the $T_{1}^{\mathrm{E}}$ state to make it closer to the $S_{1}^{\mathrm{E}}$ energy, where the energy difference is called $\Delta \varepsilon^{\prime}=E\left(\mathrm{~S}_{1}^{\mathrm{E}}\right)-E\left(\mathrm{~T}_{1}^{\mathrm{E}}\right)$. It is noted that the energy gap can be used no only as an approximation for emitter with a smaller $\Delta E_{\mathrm{S}_{1}-\mathrm{T}_{1}}$, but also where the $S_{1}$ state is close in energy to higher lying triplet states as observed for TBPe. Fig. 2d shows the FRET rate as a
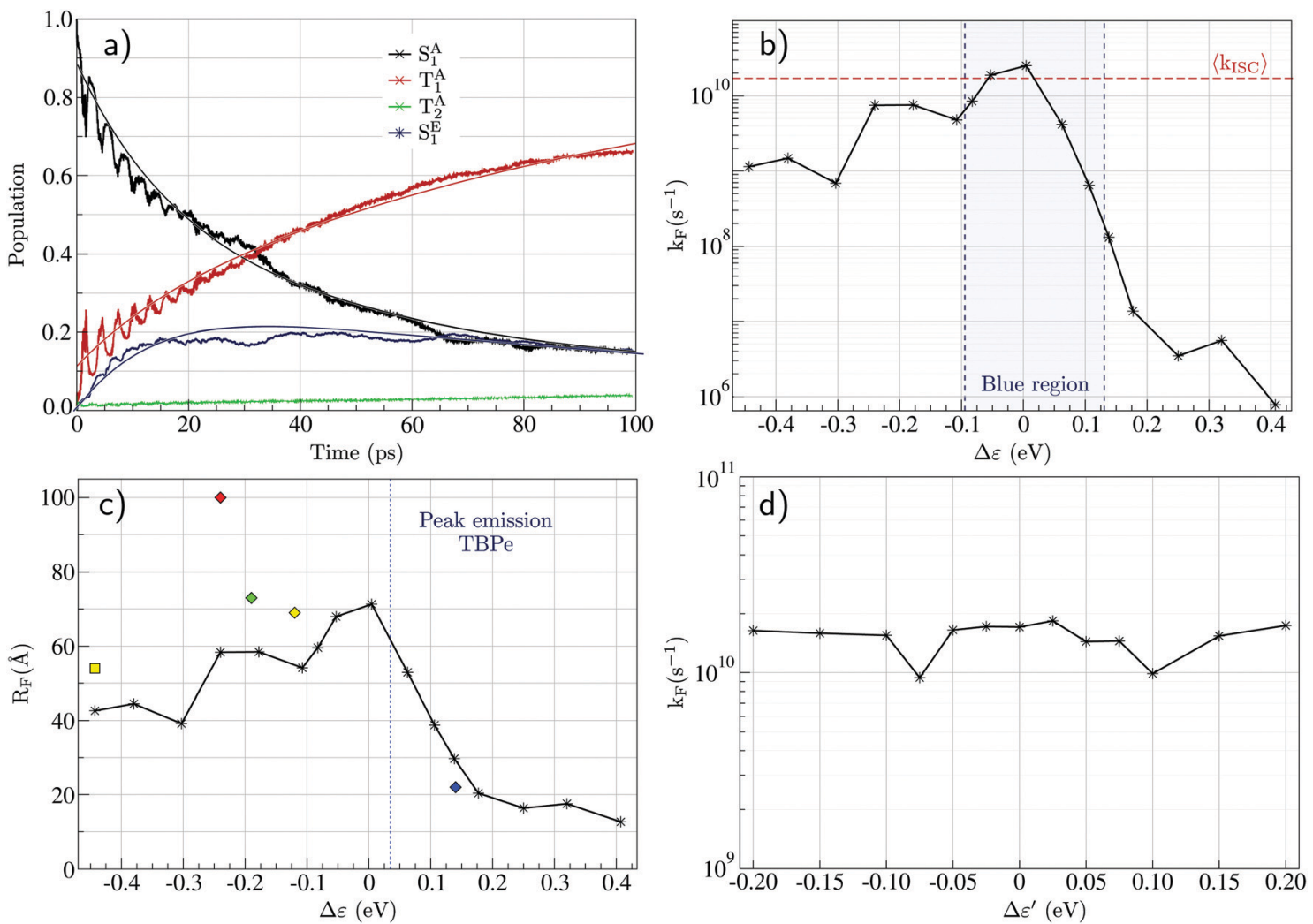

Fig. 4 (a) Evolution of the excited state populations with time for $\Delta \varepsilon=-50 \mathrm{meV}$ and $R_{\mathrm{DA}}=15.7 \AA$ (see text): $\mathrm{S}_{1}^{A}$ in black, $T_{1}^{A}$ in red, $T_{2}^{A}$ in green, and $\mathrm{S}_{1}^{\mathrm{E}}$ in blue, together with the fits (full lines) obtained from eqn (S23) (ESI†). (b) Evolution of the FRET rate constant $\left(k_{\mathrm{F}}\right)$ estimated from quantum dynamics as a function of $\Delta \varepsilon$. (c) Förster radius $\left(R_{\mathrm{F}}\right)$ calculated from eqn (S48) (ESI $\dagger$ ) as a function of $\Delta \varepsilon$ where the donor fluorescence lifetime is taken as $\tau_{\mathrm{D}}=350 \mathrm{~ns}{ }^{25}$ The diamonds are the experimental values for blue (ACRSA/TBPe), green (ACRXTN/TTPA), yellow (PXZ-TRZ/TBRb), and red (tri-PXZ-TRZ/CBP) emitters. ${ }^{13}$ The square is the experimental value for the yellow (CMA/TBRb) emitter. ${ }^{24}$ (d) Evolution of the FRET rate constant estimated from quantum dynamics as a function of $\Delta \varepsilon^{\prime}$ (see text). 
function of $\Delta \varepsilon^{\prime}$. This shows that even with the large SOC of the TADF emitter, the fluorescence emitter and the small $\Delta \varepsilon^{\prime}$ this does not give rise to a significant amount of population transfer into the triplet states, which would be detrimental. Consequently, the predominant mechanism for formation of $\mathrm{T}_{1}^{\mathrm{E}}$ would be by direct charge recombination.

\section{Discussion and conclusions}

In this work, we have used quantum chemistry and quantum dynamics simulations to study a hyperfluorescence-based system composed of a TADF triplet harvester and a narrow organic fluorescent emitter with the objective of providing a detailed understanding on the competing mechanisms involved. The quantum chemistry simulations show that in the energy range of interest it is not only the lowest singlet and triplet excited states associated with each of the isolated molecules that are present. Intermolecular charge transfer states, where the electron (or hole) is trapped on the TADF co-host and the other charge on the fluorophore can be formed and lie in close proximity to the singlet and triplet states of the donor and acceptor. However, these states can be shifted to higher in energy by increasing the separation between the donor and acceptor. The absence of these states is strongly preferable as their formation has been implicated as a loss pathway in hyperfluorescence, ${ }^{19}$ by assisting the formation of triplet states on the fluorophore.

Our quantum dynamics simulations find a typical FRET rate of $\sim 10^{10} \mathrm{~s}^{-1}$, indicating that it occurs on the picosecond timescale and is competitive with ISC. This rate yields Förster radii in quantitative agreement with previous experiments studying hyperfluorescence, ${ }^{13,24}$ indicating that the quantum dynamics can be used as a tool to understand the key factors controlling the HF mechanism. Despite this our FRET rate is substantially smaller than reported by Yang et al., ${ }^{24}$ who reported that FRET on a similar system occurs in 300 fs. In this case, it would be significantly faster than usually encountered for FRET in OLEDs, ${ }^{45}$ which is expected to occur on the 100 s of picoseconds to 10 s of nanoseconds, as found in this work. In addition, as the ISC occurs in $\sim 10 \mathrm{ps,} \mathrm{this} \mathrm{would}$ suggest that during photoexcitation negligible amount of triplet states are formed. However, the authors report a triplet population and use it to follow the lifetime of the rISC followed by FRET step which is $\sim 11 \mathrm{~ns}$. This suggest that further spectroscopic studies are required to clarify the exact nature of FRET involving Au-Cz.

It is worth stressing that the fast ISC in Au-Cz means that it represents an excellent test case for HF. Indeed, for most organic molecules the ISC rate is at least an order of magnitude slower meaning that FRET will more frequently outcompete ISC. In this scenario photoexcitation of the TADF co-host will lead directly to FRET, without populating the triplet state. This is important for studying the HF mechanism using photoexcitation, as it enables the excited TADF emitter to relax into the triplet states to undergo rISC followed by FRET in a mechanism more closely related to that obtained from electro-excitation.
In summary, our quantum dynamics have provided insight into the HF mechanism. This has highlighted the relative importance of the $\Delta \varepsilon$ and $\Delta \varepsilon^{\prime}$ energy gaps, the molecular vibrations, namely the torsional angle of $\mathbf{A u}-\mathbf{C z}$ responsible for promoting its radiative rate and enhancing FRET and the dependence of the distance between the donor and acceptor units for controlling the presence of low lying intermolecular CT states. Indeed, it is $\Delta \varepsilon$ which is most important to the efficient of HF. In contrast the triplet state of the fluorescent emitter plays very little role, even when the coupling is increased. This suggests that the formation of this state, a detrimental loss pathway, will predominantly occur through direct charge recombination. Further work should focus on increasing the spectral overlap between the $\mathbf{A u}-\mathbf{C z}$ based donor and blue emitter, which would be best achieved by increasing the emission energy of $\mathbf{A u}-\mathbf{C z}$ and increase the FRET rate. However, it is stressed that as the typical FRET rate is $\sim 10^{10} \mathrm{~s}^{-1}$, the ISC and rISC on the TADF emitter remain the rate limiting step and needs to be enhanced to improve the efficiency of HF.

\section{Conflicts of interest}

There are no conflicts to declare.

\section{Acknowledgements}

We acknowledge the EPSRC, Projects EP/P012388/1, EP/ T022442/1 and EP/R021503/1 for funding. Data supporting this publication is openly available under an Open Data Commons Open Database License? Additional metadata are available at: 10.25405/data.ncl.12752555. Please contact Newcastle Research Data Service at rdm@ncl.ac.uk for access instructions.

\section{References}

1 L. J. Rothberg and A. J. Lovinger, J. Mater. Res., 1996, 11, 3174-3187.

2 M. Baldo, D. O'brien, M. Thompson and S. Forrest, Phys. Rev. B: Condens. Matter Mater. Phys., 1999, 60, 14422.

3 C. Adachi, M. A. Baldo, M. E. Thompson and S. R. Forrest, J. Appl. Phys., 2001, 90, 5048-5051.

4 D. Y. Kondakov, Philos. Trans. R. Soc., A, 2015, 373, 20140321.

$5 \mathrm{H}$. Yersin, Highly efficient OLEDs with phosphorescent materials, John Wiley \& Sons, 2008.

6 A. Endo, M. Ogasawara, A. Takahashi, D. Yokoyama, Y. Kato and C. Adachi, Adv. Mater., 2009, 21, 4802-4806.

7 C. Adachi, Jpn. J. Appl. Phys., 2014, 53, 060101.

8 F. B. Dias, T. J. Penfold and A. P. Monkman, Methods Appl. Fluoresc., 2017, 5, 012001.

9 M. Y. Wong and E. Zysman-Colman, Adv. Mater., 2017, 29, 1605444.

10 J. Föller and C. M. Marian, J. Phys. Chem. Lett., 2017, 8, 5643-5647. 
11 T. Penfold, F. Dias and A. P. Monkman, Chem. Commun., 2018, 54, 3926-3935.

12 J. Eng and T. J. Penfold, Chem. Rec., 2020, 20, 831-856, DOI: 10.1002/tcr.202000013.

13 H. Nakanotani, T. Higuchi, T. Furukawa, K. Masui, K. Morimoto, M. Numata, H. Tanaka, Y. Sagara, T. Yasuda and C. Adachi, Nat. Commun., 2014, 5, 4016.

14 Y. J. Kang, J. H. Yun and J. Y. Lee, Org. Electron., 2020, 78, 105604.

15 J. S. Jang, S. H. Han, H. W. Choi, K. S. Yook and J. Y. Lee, Org. Electron., 2018, 59, 236-242.

16 S. H. Han and J. Y. Lee, J. Mater. Chem. C, 2018, 6, 1504-1508.

17 N. Aizawa, S. Shikita and T. Yasuda, Chem. Mater., 2017, 29, 7014-7022.

18 M. Jakoby, B. S. Richards, U. Lemmer and I. A. Howard, Phys. Rev. B, 2019, 100, 045303.

19 S. Gottardi, M. Barbry, R. Coehoorn and H. Van Eersel, Appl. Phys. Lett., 2019, 114, 073301.

20 I. Lyskov, M. Etinski, C. M. Marian and S. P. Russo, J. Mater. Chem. C, 2018, 6, 6860-6868.

21 T. J. Penfold, E. Gindensperger, C. Daniel and C. M. Marian, Chem. Rev., 2018, 118, 6975-7025.

22 M. K. Etherington, J. Gibson, H. F. Higginbotham, T. J. Penfold and A. P. Monkman, Nat. Commun., 2016, 7, 1-7.

23 J. Gibson, A. P. Monkman and T. J. Penfold, ChemPhysChem, 2016, 17, 2956-2961.

24 L. Yang, V. Kim, Y. Lian, B. Zhao and D. Di, Joule, 2019, 3, 1.

25 D. Di, A. S. Romanov, L. Yang, J. M. Richter, J. P. Rivett, S. Jones, T. H. Thomas, M. A. Jalebi, R. H. Friend and M. Linnolahti, et al., Science, 2017, 356, 159-163.

26 J. Feng, L. Yang, A. S. Romanov, J. Ratanapreechachai, A.-P. M. Reponen, S. T. Jones, M. Linnolahti, T. J. Hele, A. Köhler and H. Bässler, et al., Adv. Funct. Mater., 2020, 1908715.

27 S. Shi, M. C. Jung, C. Coburn, A. Tadle, M. R. Daniel Sylvinson, P. I. Djurovich, S. R. Forrest and M. E. Thompson, J. Am. Chem. Soc., 2019, 141, 3576-3588.

28 R. Hamze, J. L. Peltier, D. Sylvinson, M. Jung, J. Cardenas, R. Haiges, M. Soleilhavoup, R. Jazzar, P. I. Djurovich and G. Bertrand, et al., Science, 2019, 363, 601-606.
29 A. S. Romanov, C. R. Becker, C. E. James, D. Di, D. Credgington, M. Linnolahti and M. Bochmann, Chem. Eur. J., 2017, 23, 4625-4637.

30 A. S. Romanov, L. Yang, S. T. Jones, D. Di, O. J. Morley, B. H. Drummond, A. P. Reponen, M. Linnolahti, D. Credgington and M. Bochmann, Chem. Mater., 2019, 31, 3613-3623.

31 J. Feng, A.-P. M. Reponen, A. S. Romanov, M. Linnolahti, M. Bochmann, N. C. Greenham, T. Penfold and D. Credgington, Adv. Funct. Mater., 2020, 2005438.

32 S. Thompson, J. Eng and T. Penfold, J. Chem. Phys., 2018, 149, 014304.

33 J. Eng, S. Thompson, H. Goodwin, D. Credgington and T. J. Penfold, Phys. Chem. Chem. Phys., 2020, 22, 4659-4667. 34 Y. Shao, Z. Gan, E. Epifanovsky, A. T. Gilbert, M. Wormit, J. Kussmann, A. W. Lange, A. Behn, J. Deng and X. Feng, et al., Mol. Phys., 2015, 113, 184-215.

35 G. t. Te Velde, F. M. Bickelhaupt, E. J. Baerends, C. Fonseca Guerra, S. J. van Gisbergen, J. G. Snijders and T. Ziegler, J. Comput. Chem., 2001, 22, 931-967.

36 C. F. Guerra, J. Snijders, G. T. te Velde and E. J. Baerends, Theor. Chem. Acc., 1998, 99, 391-403.

37 F. Weigend and R. Ahlrichs, Phys. Chem. Chem. Phys., 2005, 7, 3297-3305.

38 D. Andrae, U. Haeussermann, M. Dolg, H. Stoll and H. Preuss, Theor. Chim. Acta, 1990, 77, 123-141.

39 C. Adamo and V. Barone, J. Chem. Phys., 1999, 110, 6158-6170.

40 S. Hirata and M. Head-Gordon, Chem. Phys. Lett., 1999, 314, 291-299.

41 A. Klamt and G. Schürmann, J. Chem. Soc., Perkin Trans. 2, 1993, 799-805.

42 C.-P. Hsu, Z.-Q. You and H.-C. Chen, J. Phys. Chem. C, 2008, 112, 1204-1212.

43 M. H. Beck, A. Jäckle, G. A. Worth and H.-D. Meyer, Phys. Rep., 2000, 324, 1-105.

44 J. Eng, B. A. Laidlaw and T. J. Penfold, J. Comput. Chem., 2019, 40, 2191-2199.

45 H. Fukagawa, T. Shimizu, Y. Iwasaki and T. Yamamoto, Sci. Rep., 2017, 7, 1-8. 